\title{
"A Comparison of General Anaesthesia Techinques Using Proseal Laryngeal Mask Airway Or Endotracheal Tube, In Patients Undergoing Laproscopic Surgery".
}

\author{
Malviya P.S ${ }^{1}$, Singh Kumar Manish ${ }^{2}$, Kumar Sumit ${ }^{3}$, \\ Yadav Kumar Dharmendra ${ }^{4}$ \\ MISHRA L . S ${ }^{5}$ \\ ${ }^{1}$ Professor ${ }^{2}$ P.G. Student ${ }^{3}$ Lecturer ${ }^{4}$ Lecturer, ${ }^{5}$ Professo Dept. Of Anaesthesiology\& Critical Care, M.L.N. \\ Medical College, Allahabad
}

\begin{abstract}
:
Introduction: Traditional open surgeries are now being done by minimally invasive keyhole laparoscopic techniques. Simultaneously, airway management of patients has also progressed from ventilation through endotracheal tube to lesser invasive Laryngeal Mask Airway. The Proseal Laryngeal Mask Airway, the latest in the family of Laryngeal Mask Airway, differs from the standard Laryngeal Mask Airway in having a drain tube in addition to a reinforced airway tube. It is a highly satisfactory device in securing an airway, it's lacunae with positive pressure ventilation (PPV), especially in patients with decreased pulmonary compliance prompted him further to find a better airway device.It also offered protection against regurgitation and gastric insufflation.

AIMS And Objective: The present study aims to compare safety and efficacy of giving general anesthesia using proseal laryngeal mask airway in patients undergoing laproscopic surgery. The above will be reached by observing vital parameters - heart rate, blood pressure, oxygen saturation, end tidal carbon di-oxide level \& any other complications as observed .
\end{abstract}

Material And Methods: The proposed study was carried out in S.R.N. Hospital associated with M.L.N. Medical College , Allahabad. After randomization \& blinding, patients will be allocated in one of the following groups. each group containing 30 patients.

Group A: Undergoing general anaesthesia using Proseal laryngeal mask Airway(PLMA).

Group B: Undergoing general anaesthesia using Endotracheal tube(ETT).

Results: Compared to PLMA group, patient that were intubated with E.T. tube showed significantly increase in Pulse rate, SBP $D B P \& M A P$ which was statistically significant till $5^{\text {th }}$ min of induction $(p$ value $<0.0001)$ \& immediate postoperative period.Postoperative there was significant increase in incidence of sorethroat in ETT group.

Conclusion: The comparative study showed PLMA has good hemodynamic stability, adequate ventilation and less post-operative airway complication. Hence, it is concluded PLMA is suiFigure and a safe alternative to cuffed ETT for airway management in elective non obese patients undergoing laparoscopic surgeries under general anaesthesia

Keywords: Minimally invasive Keyhole laparoscopic techniques, Proseal laryngeal mask Airway, General anaesthesia

\section{Introduction}

General anaesthesia with Intermittent Positive Pressure Ventilation in cases undergoing laparoscopic surgery poses special problem. Due to insufflation of carbon di oxide inside abdomen, the rise of intraabdominal pressure and also splinting of diaphragmatic movement occurs. General anesthesia with cuffed endotracheal tube effectively maintains ventilation and protects respiratory tract against regurgitation and inhalation of gastric contents.

Laryngeal mask airway (LMA) is a supraglottic airway device, is a highly satisfactory device in securing an airway, it's lacunae with positive pressure ventilation (PPV), especially in patients with obesity and decreased pulmonary compliance prompted him further to find a better airway device. Proseal Laryngeal Mask Airway (PLMA), the latest in the family of LMA, developed in the late 1990's with improved ventilatory characteristics. It also offered protection against regurgitation and gastric insufflation. ${ }^{1}$

Laparoscopic surgeries are day care surgery because it is minimally invasive surgery, So theairway management of the patients undergoing laparoscopic procedures has progressed from Endotracheal intubation (ETT) to lesser invasive devices like Proseal Laryngeal Mask Airway (PLMA). ${ }^{2}$ Disadvantages of tracheal intubation includes intra-operative hemodynamic changes, post-operative complication like hoarseness of voice 
and sore throat. SoProseal Laryngeal Mask Airway has challenged the standard Endotracheal tube used during general anesthesia. Therefore this study is undertaken to compare the efficacy of Proseal Laryngeal Mask Airway over Endotracheal tube in patients undergoing laparoscopic surgery under general anesthesia with controlled ventilation.

\section{Material \& Methods}

The present study was conducted in Department of Anaesthesiology and Critical Care Medicine, M.L.N. Medical College Allahabad.ASA grade I \& II patients of either gender scheduled to undergo elective laparoscopic surgeries under general anaesthesia were included in the prospective randomized study after approval from ethical committee, and informed consent from patient. The patients between 25-60 yrs of age were randomly allotted through blinding to either PLMA or ETT group for airway management.

After a thorough pre-anestheticcheckup, all selected patients were subjected for routine and specific investigations, and instructed to remain fasting for 8 hours prior to surgery and written informed consent for general anesthesia was taken.On arrival in the operating room, the multipara monitor was attached and the vital parameters were recorded including, patient's pulse rate, noninvasive blood pressure, and oxygen saturation. A large bore intravenous cannula was inserted for drugs and fluid administration.

Patients undergoing study were divided into two groups:-

Group-A Patients whose airway was managed with Proseal Laryngeal Mask Airway (PLMA)

Group-B Patients whose airway was managed with cuffed Endotracheal tube (ETT)

All the patients of Group A and Group B were premedicated with intra venous midazolam $0.1 \mathrm{mg} / \mathrm{kg}$, glycopyrrolate $0.2 \mathrm{mg} \mathrm{I.V.,} \mathrm{fentanyl} 1 \mathrm{mcg} / \mathrm{kg}, 5 \mathrm{~min}$ prior to induction. Patients of Group A and Group B were preoxygenated with 100\% oxygen for 3 minutes and then induced with injection $1 \%$ propofol $2 \mathrm{mg} / \mathrm{kg}$ slow intra venous till the loss of verbal command, and placement of airway device facilitated with rocuronium 1 mg/kg I.V. In Group A, an appropriate size PLMA (LMA-PROSEAL ${ }^{\mathrm{TM}}$ Laryngeal Mask Company, (U.K.) Limited, HP10 0HH, UK) was inserted as per recommendations based on weight criteria; i.e., size 3 for patients weighing $30-50 \mathrm{~kg}$ and size 4 for $50-70 \mathrm{~kg}$.

Group B - Cuffed Endotracheal tube (7.5 in females and 8.5 in males) was placed, using Mac'intosch curved laryngoscope blade.Time taken to insert and number of attempts for placing the device was noted.

The time interval between holding the airway device to confirmation of correct placement by bilateral air entry on chest auscultation was noted. Anaesthesia was maintained with nitrous oxide and oxygen (60:40), isoflurane $(1-1.5 \%)$ and vecuronium $(0.03 \mathrm{mg} / \mathrm{kg})$ and ventilation was maintained with tidal volume $8 \mathrm{ml} / \mathrm{kg}$ , fraction of inspired oxygen $\left(\mathrm{FiO}_{2}\right) 0.33$, respiratory rate of $12 / \mathrm{min}$ and $\mathrm{I} / \mathrm{E}$ of $1: 2$. The $\mathrm{FiO}_{2}$ and respiratory rate were adjusted to maintain $\mathrm{SpO}_{2}>95 \%$ and $\mathrm{EtCO}_{2}(35-45) \mathrm{mm} \mathrm{Hg}$ (i.e. in normal range). Intra-abdominal pressure (IAP) was maintained $<14 \mathrm{~mm} \mathrm{Hg}$. The Ryle's tube of appropriate size $14 \mathrm{~F}$ in group A and $16 \mathrm{~F}$ in group B was placed in all the patients.After completion of surgery, the adequate neuromuscular blockade reversal was achieved with glycopyrrolate $(0.01 \mathrm{mg} / \mathrm{kg})$ and neostigmine $(0.05 \mathrm{mg} / \mathrm{kg})$.

\section{The Following Parameters Were Recorded:}

- Insertion characters

- No of attempts for insertion of device,

- Hemodynamic respiratory responses-:

- $\quad$ PR, Systolic and Diastolic BP, MAP, $\mathrm{SpO}_{2}$ was recorded at following intervals:

- Baseline,

- During insertion of device at 1 minute, 3 minutes, 5 minutes and 15 minutes,

- After removal of device.

- $\mathrm{EtCo}_{2}$ was recorded at following intervals:

- During insertion of device at 1 minute, 3 minutes, 5 minutes and 15 minutes,

- Incidence of oesophageal regurgitation, gastric insufflation, pulmonary aspiration and displacement of device were noted.

- Postoperative incidence of blood stained secretion, sorethroat, dysphasia, dysphonia, and trauma to lip and teeth were noted. 
III. Observation \& Results

Figure 1 :-Details of Airway Insertion

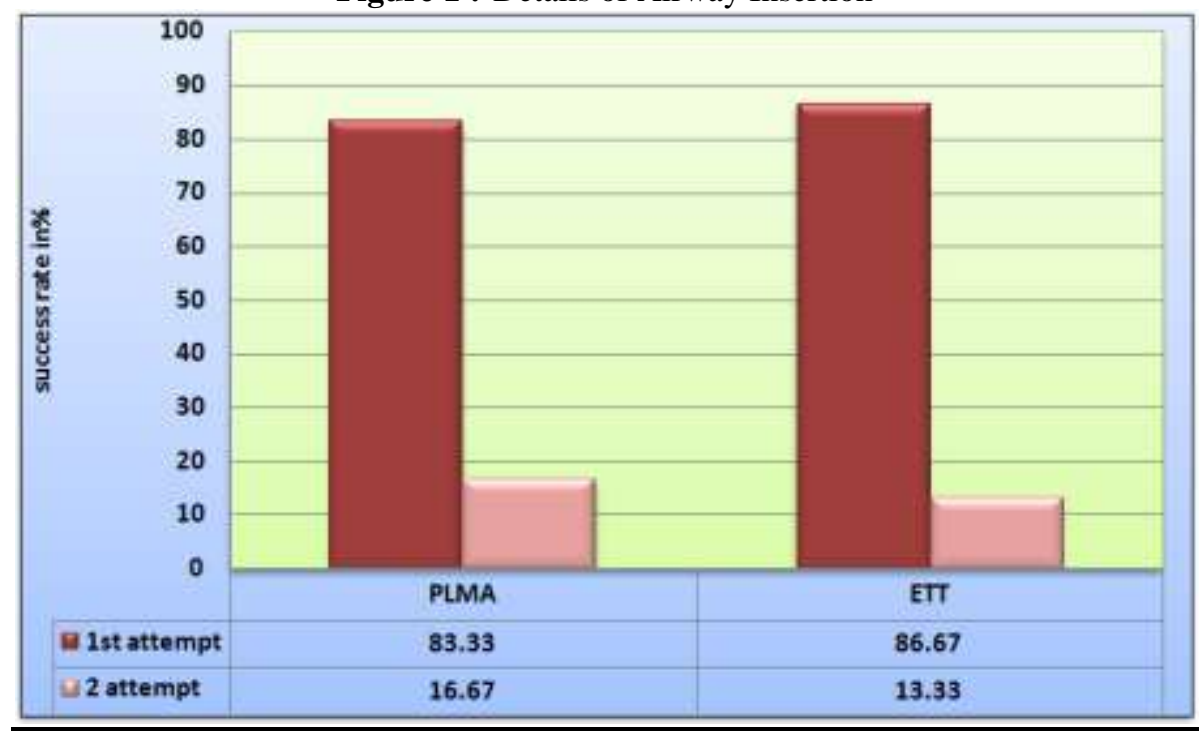

Figure -2: Comparison of Pulse Rate on insertion

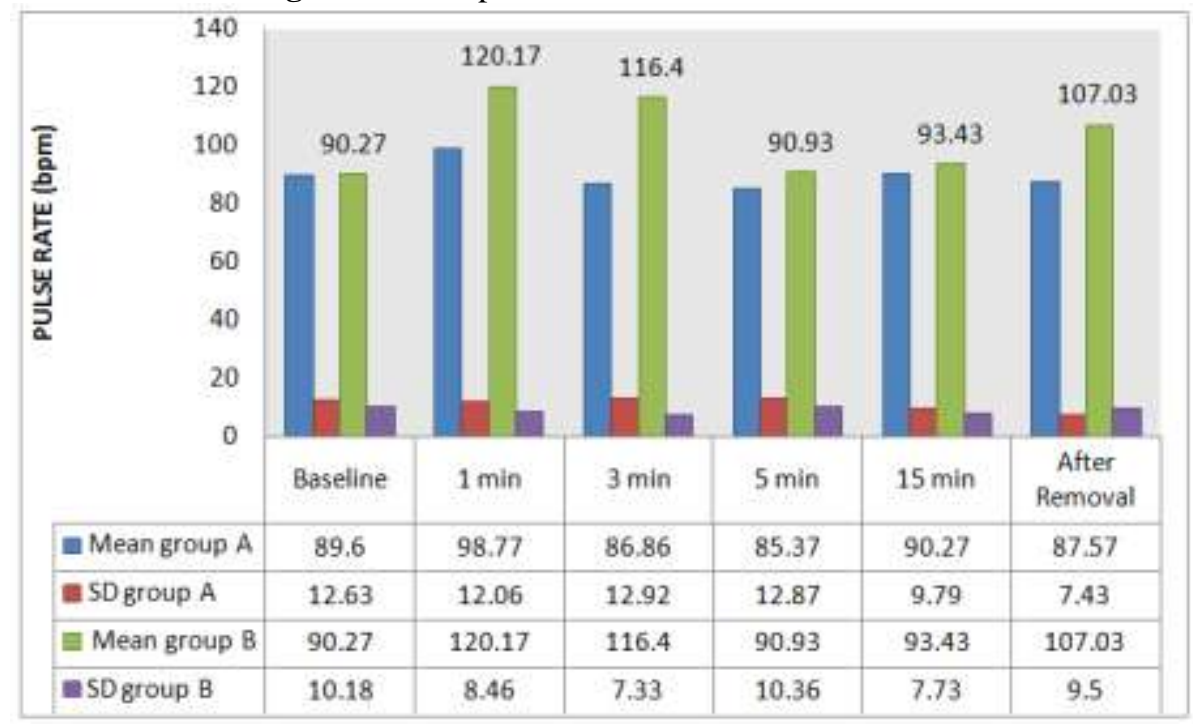

Figure -3: Comparison of SBP after

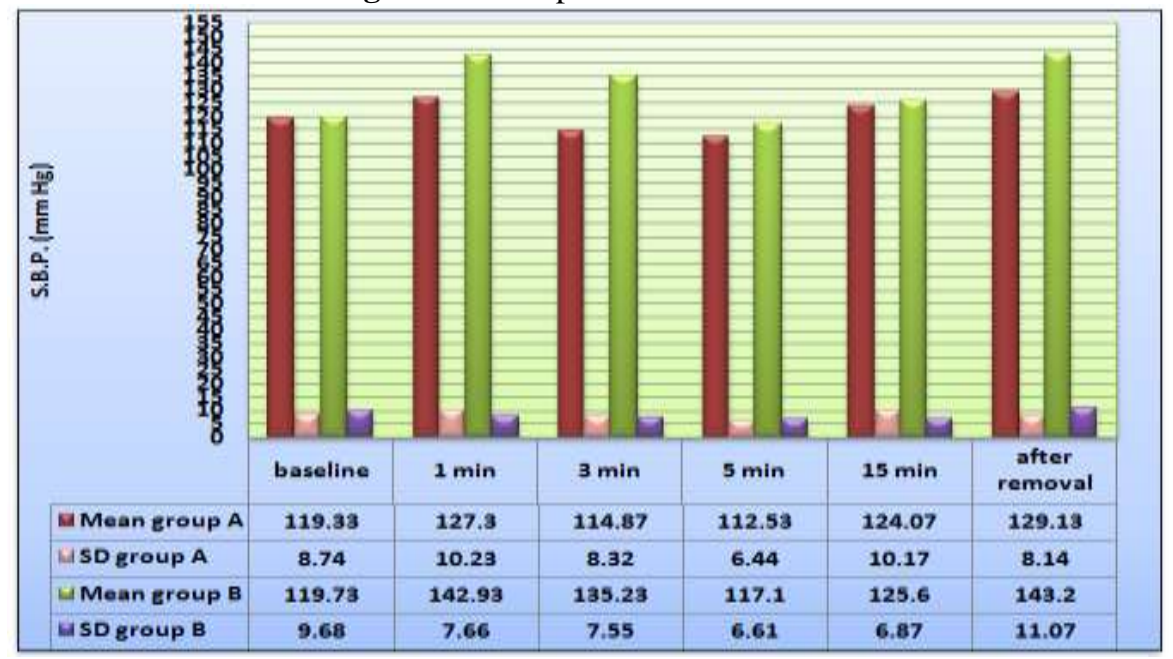

Figure -4: Comparison of Diastolic Blood Pressure 


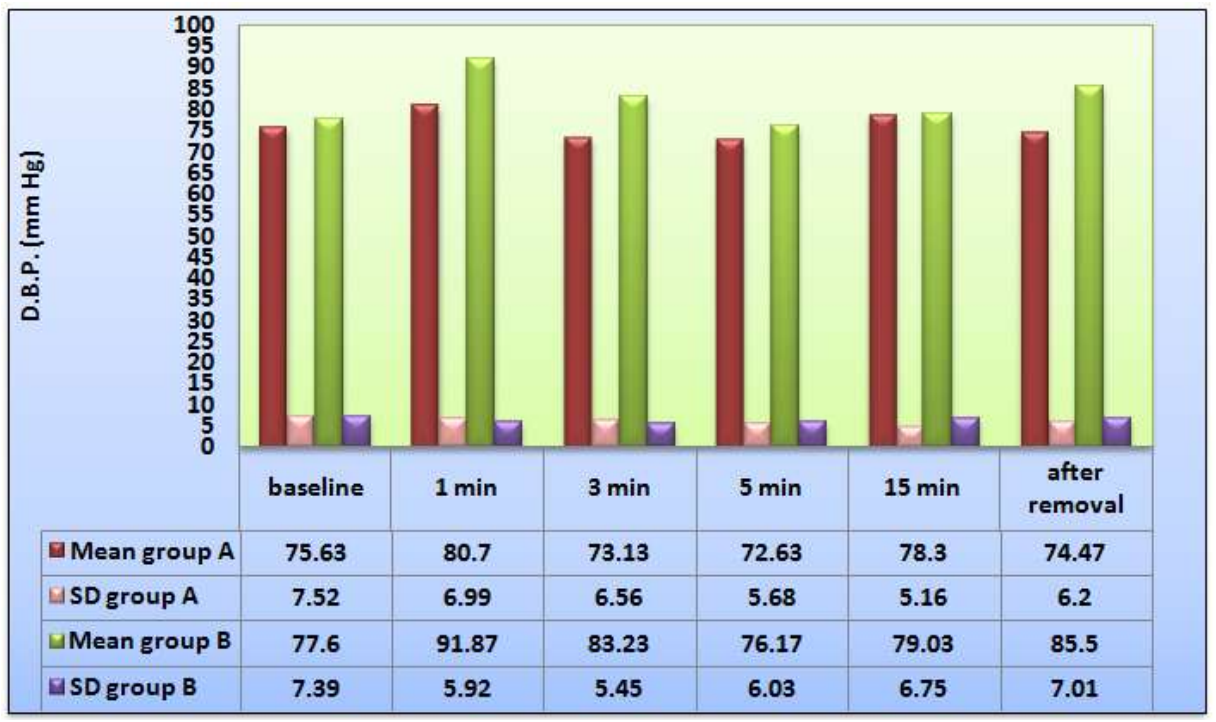

Figure 5: Comparison of Mean Arterial Pressure

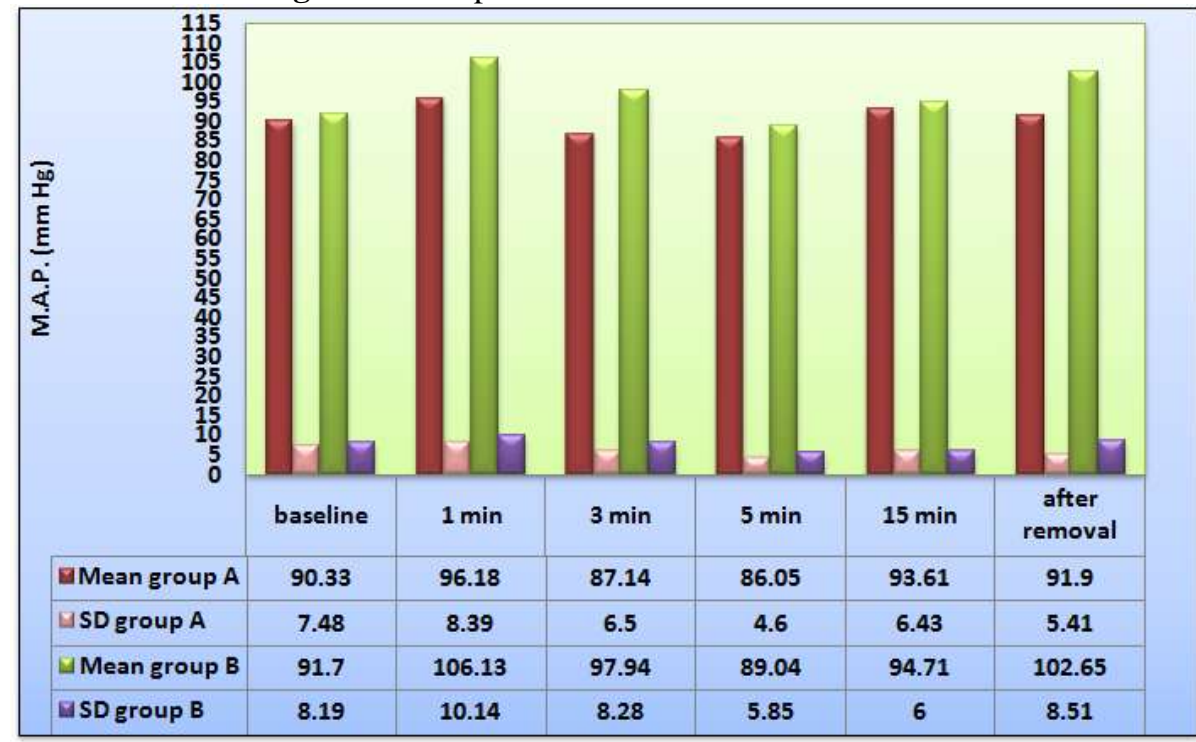

Figure 6 : Comparison of Changes In $\mathrm{EtCO}_{2}$

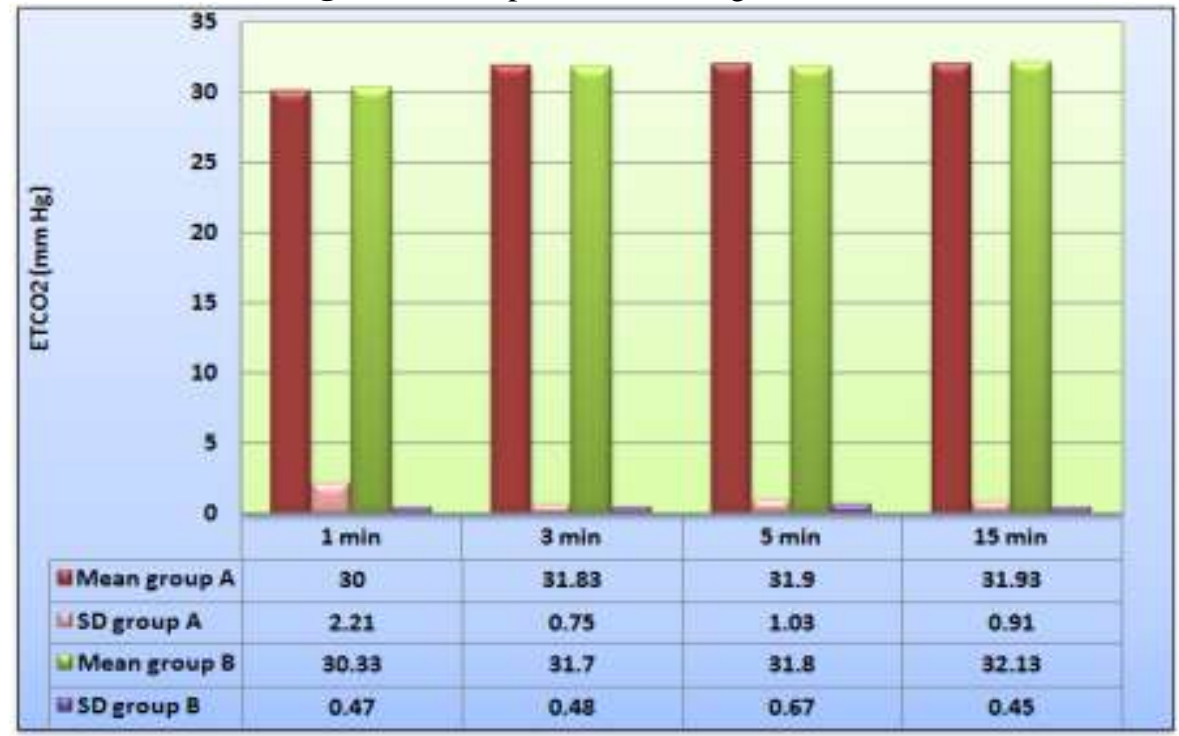

Figure 7 : Comparison of Changes In $\mathrm{SpO}_{2}$ 


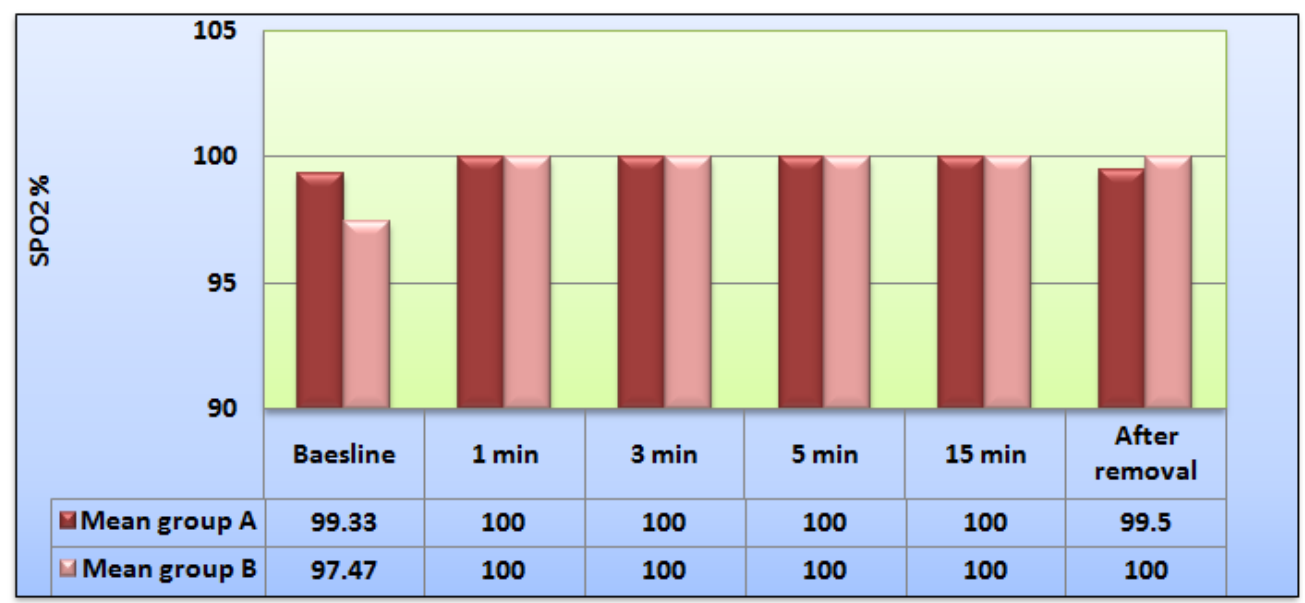

Figure 8 :- Adverse Events

\begin{tabular}{|c|c|c|c|}
\hline & Group A & Group B & P value \\
\hline \multicolumn{4}{|l|}{ Intraoperative } \\
\hline A) Oropharyngeal leak & - & - & \\
\hline B) Gastric insufflation & 6 & - & \\
\hline C) Esophageal regurgitation & - & - & \\
\hline D) Pulmonary aspiration & - & - & \\
\hline E) Displacement of device & 3 & - & \\
\hline \multicolumn{4}{|l|}{ Removal } \\
\hline A) Coughing & 2 & 6 & \\
\hline B) Blood staining of Device & & 6 & \\
\hline C) Vomiting & - & - & \\
\hline D) Regurgitation & - & - & \\
\hline E) Laryngospasm & - & - & \\
\hline F)Trauma; lip \& teeth & 2 & 4 & \\
\hline \multicolumn{4}{|l|}{ Postoperative } \\
\hline A) Sorethroat & 1 & 5 & \\
\hline B) Dysphagia & - & - & - \\
\hline C) Dysphonia & - & - & - \\
\hline
\end{tabular}

\section{Discussion}

In an attempt to test the efficacy and safety of PLMA as a airway device over ETT for ventilation, for a variety of commonly performed laparoscopic surgeries, the present study was conducted following institutional committee approval and written informed consent, on 60 patients of either sex ,aged 25 to $60 \mathrm{yrs}$ ,ASA physical status 1 and 2 scheduled for elective laproscopic surgery and all the patient under study randomly were divided in two groups, GROUP A (PLMA ) and GROUP B (ETT) of 30 each.The demographic data, i.e age, weight was comparable in 2 groups. Mean age $34.53 \pm 6.25$ years in group A, while it was $33.57 \pm 6.76$ years in group B, and hence comparable. Mean weight distribution of patients $58.83 \pm 6.3 \mathrm{~kg}$ in group A, while it was $57.17 \pm 5.44 \mathrm{~kg}$ in group B . Most of patients in both groups were operated for laproscopic cholecystectomy.

The PLMA is a new entrant to the family of LMA with some added features over the classic LMA, PLMA can be inserted using either the introducer, index finger or the thumb. For the purpose of standardization, we used the introducer for all the cases. Size 4 PLMA placement was attempted in all patients. Insertion success rate was $83.33 \%$ for the first attempt, and two attempts were made in $16.66 \%$ patients. In Group B, the insertion success rate was $86.67 \%$ for the first attempt; two attempts were taken in $13.33 \%$ of patients. There was no third attempt or failed insertion in either group.Our results are in accordance to that of NamitaSaraswatiet al $\mathbf{l}^{3}$ who reported Insertion success rate was $86.67 \%$ for the first attempt, and $13.33 \%$ in two attempts. Insertion was easy in 23 and difficult in 7 patients. In Group B, the insertion success rate was $83.37 \%$ for the first attempt; two attempts were made in $13.33 \%$ of patients and third attempt was required in $3.33 \%$ patients, in each group in their study comparing between PLMA and ETT for laparoscopic surgery under general anaesthesia. These difference may be because of neuromuscular blockade was achieved with vecuronium bromide for intubation in above study, While in our study; it was rocuronium which provide excellent relaxation.

M.N. Mishra, B. Ramamurthy ${ }^{1}$, reported that all the patients in the tracheal tube group were subjected to intubation successfully at first attempt $(100 \%)$. There was no need for further attempts in this group. On the other hand, PS-LMA group had 88\% (44/50) first attempt success at insertion $(\mathrm{P}<0.01)$

Hemodynamic parameters like pulse rate wasincreased in both groups from the baseline values during insertion of device, but statistically significant increase in PR was noticed in Group B at $1^{\text {st }}$ min, $3^{\text {rd }}$ min which 
lasted upto $5^{\text {th }}$ min from insertion of device in comparison to Group A there was no significant difference in increase in pulse rate after 15 minutes between groups. Pulse rate was increased after removal of device in both groups, but it was statistically significantly higher in group B $(\mathrm{p}<0.0001)$.

On comparing trends within group, increase in pulse rate was observed 1 minute after intubation and persisted till 3 minutes and after extubation in both group. But this increase was highly significant in group B $(\mathrm{p}<0.0001)$.

Systolic blood pressure was increased in both Groups from baseline values during insertion of device, but statistically significant increase in SBP was noticed in Group B at $1^{\text {st }} \min , 3^{\text {rd }} \min (\mathrm{p}<0.0001)$ and it lasted upto $5^{\text {th }} \mathrm{min}(\mathrm{p}<0.006)$ from insertion of device in comparison to Group A. SBP was increased after removal of device in both groups, but it was statistically significantly higher in group B $(p<0.0001)$.

On comparing trends within groups, statisticallysignificantly higher $(\mathrm{p}<0.0001)$ increase in SBP was observed at 1 minute after intubation and persisted till 3 minutes and after extubation in group B, however, statistically significant increase in SBP in group A was seen only at $1 \mathrm{~min}$ after insertion of device.

Diastolic blood pressure was increased in both Groups from baseline values during insertion of device, but statistically significant increase in DBP was noticed in Group B at $1^{\text {st }} \min , 3^{\text {rd }} \min (\mathrm{p}<0.0001)$ and it lasted upto $5^{\text {th }} \min (\mathrm{p}<0.0193)$ from insertion of device in comparison to Group A. there was no significant difference in increase in DBP after 15 minutes between groups. DBP was increased after removal of device in both groups, but it was statistically significantly higher in group B $(\mathrm{p}<0.0001)$.

On comparing trends within groups, statisticallysignificantly higher $(\mathrm{p}<0.0001)$ increase in DBP was observed at 1 minute after intubation and persisted till 3 minutes and after extubation in group B, however, statistically significant increase in DBP in group A was seen only at $1 \mathrm{~min}$ after insertion of device.

Mean arterial pressure was increased in both Groups from baseline values during insertion of device, but statistically significant increase in MAP was noticed in Group B at $1^{\text {st }} \min , 3^{\text {rd }} \min (\mathrm{p}<0.0001)$ and it lasted upto $5^{\text {th }} \mathrm{min}(\mathrm{p}<0.027)$ from insertion of device in comparison to Group A. There was no significant difference in increase in MAP after 15 minutes between groups. MAP was increased after removal of device in both groups, but it was statistically significantly higher in group B $(\mathrm{p}<0.0001)$.

On comparing trends within groups, statisticallysignificantly higher $(\mathrm{p}<0.0001)$ increase in MAP was observed at 1 minute after intubation and persisted till 3 minutes and after extubation in group B, however, statistically significant increase in MAP in group A was seen only at 1 min after insertion of device.

In Our study, insertion of device in Group A was associated with less hemodynamic change in comparison to Group B. Intubation and extubation was associated with significant higher increase in pulse rate and blood pressure in Group B which may further be disadvantageous in compromised patients.

Our results are similar to that of NamitaSaraswatet al $^{3}$ reported, increased in PR and increased in MAP in Group B as which was statistically significant till $3 \mathrm{~min}$, as compared to hemodynamic parameters in Group A.

Our results are in accordance to Arfatet $\mathbf{A l}^{4}$ who noticed a exaggerated hemodynamic response in terms of increased PR and MAP, at 1, 3, 5 minute after induction.

M.N. Mishra, B. Ramamurthy (2008) $)^{I}$ noticed a significant rise in HR and MAP in both the PS-LMA $(\mathrm{P}<0.05)$ and the TT groups $(\mathrm{P}<0.01)$ from their baseline values during insertion of the respective devices. On comparing the degree of rise in HR and MAP between the groups, TT group showed a higher rise in these parameters than P-LMA group $(\mathrm{P}<0.05)$.

Suboptimal oxygenation defined $\mathrm{as}_{\mathrm{SpO}_{2}}$ between $90-95 \%$ and hypoxiaas $\mathrm{SpO} 2<90 \%$. Optimal oxygenation was noted in all cases before and after $\mathrm{CO}_{2}$ - insufflation. All patients had $\mathrm{EtCO}_{2}(35-45) \mathrm{mm} \mathrm{Hg}$ after $\mathrm{CO}_{2}$ - insufflation. Our study result are similar to that of NamitaSaraswatet al ${ }^{3}$ who also reported no change in $\mathrm{SpO}_{2}$ and $\mathrm{EtCO}_{2}$ comparing with ETT in laparoscopic surgeries under general anaesthesia.

Sharma et $\boldsymbol{a l} \boldsymbol{l}^{5}$ and Maltby $\boldsymbol{e t} \boldsymbol{a l}^{\boldsymbol{6}}$ reported no statistically significant differences in $\mathrm{SpO}_{2}$ or $\mathrm{EtCO}_{2}$ between the two groups before or during peritoneal insufflations.

Minimal gastric distension, which was noticed by surgeon, was seen in 6/30(20\%) patients of Group A. This however did not disturb the field of vision and was not quantitatively measured. But none was seen in Group B. There was displacement of PLMA in 3(10\%) patients in Group A. There was no incidence of oropharengeal leak, esophageal regurgitation, and pulmonary aspiration in both group.

Coughing after removal of PLMA was seen in $6.66 \%$ patients, while it was seen in $20 \%$ patients in the ETT group. Blood staining of device on removal was not seen in patients in group A and in 6/30(20\%) patients in group B. Minor trauma to the lip and gums was seen in 4/30 patient (13.33\%) in group B and 2/30 (6.66\%) patients in group A. Which was statistical significant. There was no incidence of regurgitation, vomiting at the time of removal of device in any patient.

There was statistically significant higher incidence of sorethroat in group B (16.66\%) in comparison to group A $(3.33 \%)$, noted within $24 \mathrm{hr}$ of post operative period. There was no incidence of dysphonia and dysphagia in both groups. 
The double cuff arrangement of the PLMA prevents the chances of aspiration. Nasogastric tube was inserted in all our cases via the drain tube, after confirming that there was no evidence of leak,. There was no evidence of regurgitation or aspiration seen as evidenced by the maintenance of saturation and the end-tidal carbon dioxide within normal limits during the entire duration of the laparoscopy.

P.Shroffet $\boldsymbol{a l}^{2}$ and Higgins $\boldsymbol{e t}^{\mathbf{a l}} \boldsymbol{l}^{7}$ found the greatest incidence of sore throat in patients undergoing intubation than in those in whom a PLMA was used. The virtual absence of sore throat in PLMA Group could be explained by the fact that it is a supraglottic device and mucosal pressures achieved are usually below pharyngeal perfusion pressures.

\section{Conclusion}

The comparative study showed Proseal Laryngeal Mask Airway has good ease of insertion, hemodynamic stability, adequate ventilation and less post-operative airway complication. Hence, it is concluded Proseal Laryngeal Mask Airway is suiFigure and a safe alternative to cuffed Endotracheal Tube for airway management in elective non obese patients undergoing laparoscopic surgeries under general anaesthesia.

\section{References}

[1] Misra MN, Ramamurthy B. The Pro-Seal LMAtm and the tracheal tube: A comparison of events at insertion of the airway device. Internet J Anesthesiol. 2008; Vol. 16

[2] P.P. Shroff, S.K Kamath. Randomized Comparative Study Between The Proseal Laryngeal Mask Airway And The Endotracheal Tube For Laparoscopic Surgery. The Internet Journal of Anesthesiology. 2006; Volume 11 Number 1.DOI:10.5580/e8e.

[3] Namita Saraswat, Aditya Kumar, Abhijeet Mishra, Amrita Gupta, Gyan Saurabh, and Uma Srivastava ;The comparison of Proseal laryngeal mask airway and endotracheal tube in patients undergoing laparoscopic surgeries under general anaesthesia, Indian $\mathbf{J}$ Anaesth. 2011 Mar-Apr; 55(2): 129-134.

[4] Arfat Waheed, MD, Abdul Hameed, MD, et all In: ProSeal laryngeal mask airway and endotracheal tube in elderly hypertensive patients undergoing routine surgical procedures: A comparison of hemodynamic parameters., Journal of Clinical Gerontology and Geriatrics sept.2013 Volume 4, Issue 3 , Pages 80-83.

[5] Bimla Sharma et al Efficacy and Safety Performance of ProsealTM Laryngeal Mask Airway in Laparoscopic Surgery: Experience of 1000 Cases. The Indian Journal of Anesthesiol.

[6] Maltby JR, Beriault MT, Watson NC, Liepert D, Fick GH, The LMA-ProSeal is an effective alternative to tracheal intubation for laparoscopic cholecystectomy Can J Anaesth. 2002 Oct;49(8):857-62

[7] Higgins PP, Chung F, Mezei G. Postoperative sore throat after ambulatory surgery. Br J Anaesth. 2002;88:582-4.

[8] Handan Güleç, Türkay Çakan,et all in feb. 2012: Comparison of hemodynamic and metabolic stress responses caused by endotracheal tube and Proseal laryngeal mask airway in laparoscopic cholecystectomy: J Res Med Sci. Feb 2012; 17(2): 148-153

[9] Cook TM, Nolan JP, Verghese C, Strube PJ, Lees M, Millar JM, et al. A randomized crossover comparison of the proseal with the classic laryngeal mask airway in unparalysed anaesthetized patients. Br J Anaesth. 2002;88:527-33. 81

[10] Seung H. Yu, BS, and O. Ross Beirne, Laryngeal Mask Airways Have a Lower Risk of Airway Complications Compared With Endotracheal Intubation: A Systematic Review10 American Association of Oral and Maxillofacial Surgeons J Oral Maxillofac Surg 68:2359-2376, 2010

[11] Soad A. Mansour, Wafaa G.Ahmed, Kawthar A. Azzam ,Tarek M. EL said. Safety And Efficacy Of Proseal Laryngeal Mask Airway Versus Classic Laryngeal Mask Airway And Endo Tracheal Tube During Elective surgery. The Egyptian Journal of Hospital Medicine Vol., 21: 82 - 94 December 2005

[12] S. Saini, S. Taxak, S. Das: Prevention of aspiration by LMA ProSeal in laparoscopic surgery. The Internet Journal of Anesthesiology. 2009 Volume 20 Number 1. DOI: 10.5580/1569

[13] Tahereh Parsa 1, Shideh Dabir 1, Badiolzaman Radpay 1,2Ventilation with ProSeal Laryngeal Mask Airway during Short-Term Elective Gynecologic SurgeryVentilation with ProSeal Laryngeal Mask Airway during Short-Term Elective Gynecologic Surgery. Tanaffos (2006) 5(3), 19- 23-2006 NRITLD, National Research Institute of Tuberculosis and Lung Disease, Iran.

[14] Hawthorne L, Wilson R, Lyons G Studied That Four Of 16 Obese Lma-Proseal Patients Were Crossed Over To Ett Because Of Respiratory Obstruction Or Airway Leak AJA- Online.com 2011;Vol 12

[15] Abdel El-Ganzouri, Michail N. Avramov, Stetan Budac, Mariomoric, Kenneth J. Tuman. Proseal Laryngeal mask airway versus endotracheal tube: Ease of insertion, Haemodynamic responses and emergence characteristics. Anesthesiology 2003;99:A571 